\title{
Atemnot und schöner Atem. Der Atem als Souveränitätspolitikum bei Marcel Duchamp
}

\begin{abstract}
Vielen seiner Zeitgenossen muss Marcel Duchamp, einer der wirkmächtigsten Künstler des 20. Jahrhunderts, in hohem Alter wie ein lebensund zugleich kunstweiser Pensionär erschienen sein. ${ }^{1}$ Obgleich, und dies hat die Duchamp-Forschung sehr tiefgreifend erschlossen, er bis zu seinem Lebensende recht umtriebig gewesen ist ${ }^{2}$ hatte Duchamp (18871968) dennoch von sich das Bild eines Grandseigneurs, eines emeritierten Künstlers, eines vorzeitigen Ruheständlers entworfen und seine angebliche Selbstemeritierung geradezu öffentlich zelebriert. ${ }^{3}$ Immer wieder stilisierte er sich in den 1950er und 60er Jahren öffentlich als ein entschieden Untätiger - und (er)fand dafür die Selbstbezeichnung als „Atmer" (breather); ${ }^{4}$ um seine Untätigkeit zu markieren, rekurrierte Duchamp auf den Atem, auf das Atmen und auf sich als "respirateur“.5

Im Herbst 1954 beispielsweise teilte Duchamp im Alter von 67 Jahren mit, er habe, statt zu arbeiten, nunmehr ,die Art zu atmen ausgeweitet". ${ }^{6}$ Ein gutes Jahrzehnt später erklärte der damals 78-Jährige, er lebe lieber, „atme lieber“"7 als dass er arbeite. Bereits im Frühjahr 1957 hatte er allgemein von Künstler*innen - damit aber auch und gerade sich selbst meinend - behauptet: „Man lebt und man weiß nicht, wie man lebt. Man stirbt einfach nicht. [...] Die Leute fragen Künstler ständig, wie sie leben. Sie brauchen nicht zu leben. Sie atmen einfach."8 Und zwei Jahre später, im November 1959, teilte Duchamp mit, er ver-
\end{abstract}

1 Der vorliegende Text geht zurück auf einen Abendvortrag mit dem Titel „Der Künstler als Respirator. Marcel Duchamps Atemarbeit“, den ich am 12. September 2019 auf der Tagung Atem. Gestalterische, ökologische und soziopolitische Dimensionen 1900-Gegenwart an der Humboldt-Universität zu Berlin gehalten habe.

2 Siehe bspw. Stefan Banz (Hg.), Marcel Duchamp and the Forestay Waterfall, Zürich 2010.

3 Siehe hierzu insb. Lars Blunck, Duchamps Readymade, München 2017, S. 210-228.

4 Serge Stauffer (Hg.), Marcel Duchamp. Interviews und Statements, Ostfildern-Ruit 1992, S. 85, engl.: Anonym, „Art Was a Dream“, in: Newsweek 9 (54), New York, 9. November 1959, S. 118-119, hier: S. 119: „breather“.
5 Stauffer 1992 (wie Anm. 4), S. 85, engl.: Newsweek 1959 (wie Anm. 4), S. 119.

6 Ebd., S. 47, frz.: Alain Jouffroy, „Conversations avec Marcel Duchamp“ [1961], in: ders. (Hg.), Une Révolution du regard. A propos de quelques peintres et sculpteurs contemporains, Paris 1964, S. 107-124, hier: S. 109: „ J'ai élargi la manière de respirer."

7 Pierre Cabanne, Gespräche mit Marcel Duchamp, Köln 1972 [frz. 1967], S. 108, frz.: Pierre Cabanne, Entretiens avec Marcel Duchamp, Paris 1967, S. 134: „J'aime mieux [...] respirer."

8 Stauffer 1992 (wie Anm. 4), S. 64 [Übers. geänd.], engl.: Geoffrey Hellman, „Marcel Duchamp“, in: The New Yorker, 6. April 1957 (Fotokopie, Serge Stauffer Archiv, Staats- 
bringe seine Zeit mit „Nicht-Malen“, und führte aus: „Ich glaube, man könnte sagen, ich verbringe meine Zeit mit Atmen [...]. Ich bin ein respirateur - ein Atmer. Ich genieße das ungeheuerlich. “9 Mithin gab Duchamp vor, nicht (mehr) zu arbeiten, anspruchslos zu leben, eine geradezu Bartleby'sche Untätigkeit zu pflegen und es schlichtweg beim Atmen zu belassen. Er ging sogar so weit, das Atmen als „eine Art beständige Hochstimmung “ (une sorte d'euphorie constante) ${ }^{10}$ auszuweisen. Vielleicht könnte man mit Jacques Rancière auch von „eine [r] ununterbrochene[n] Gegenwart" ${ }^{\text {"11 }}$ sprechen, von einem „Glück des Nichtstuns“, ${ }^{12}$ von einer „Empfindung des Daseins"13. Für Duchamp jedenfalls manifestierte sich ein solches Dasein im geradezu subliminalen Akt des Atmens: „[J]eder Atemzug“, so führte er 1966 aus, „ist ein Werk, das nirgends registriert wird, das weder visuell noch zerebral ist" .14

So gesehen wäre Atmen die Tätigkeit des Untätigen. Und tatsächlich fasste Duchamp, wie ich in meinem Buch Duchamps Readymade ${ }^{15}$ dargelegt habe, das Atmen sogar als eine Form künstlerischer Praxis auf. Eine Auffassung, die ganz in der Tradition einer seiner in den 1910er Jahren notierten künstlerischen Kerngedanken stand: „Kann man Werke machen, die nicht ,Kunst" sind?“ (Peut-on faire des auvres qui ne soient pas , d'art'?). ${ }^{16}$ Atmen war für Duchamp selbstredend keine ,Kunst“ (im Sinne von Hochkunst), wohl aber ein Werk (also das ,Machen“ eines Künstlers): ein Werk, das sich als unterschwellige "Atemarbeit "17 verrichtete und das man - wie beim Readymade - bloß auszuwählen brauchte, um damit etwas zu machen. Dergestalt konnte

galerie Stuttgart), S. 25-27, hier: S. 26: „You live and you don't know how you live. You just don't die. [...] People ask artists always how they live. They don't have to live. They just breathe."

9 Stauffer 1992 (wie Anm. 4), S. 85, engl.: Newsweek 1959 (wie Anm. 4), S. 119: "not painting“; „I suppose you could say I spend my time breathing. [...] I'm a respirateur - a breather." Zur Funktion und Geschichte von Respiratoren siehe u.a. A. Barrington Baker, „Artificial Respiration. The History of an Idea“, in: Medical History 4 (15), 1971, S. 336-351; Claudia Schmitt, Rettung und Wiederbelebung Verunglückter, 1740-1840. Mit besonderer Berücksichtigung der Atmungs- und Beatmungsgeräte sowie anderer Hilfsmittel, Frankfurt am Main u.a. 2012; Hans Christian Niggebrügge, Die Geschichte der Beatmung - Analyse und Neubewertung am Beispiel der Geschichte des „Pulmotor" Notfallbeatmungs- und Wiederbelebungsgeräts der Lübecker Drägerwerke, Dissertation an der Universität Lübeck 2011, S. 9-108 (verfügbar unter: http://www.zhb.uni-luebeck.de/epubs/ediss1082.pdf, letzter Zugriff: 8.4.2020).

10 Cabanne 1972 [1967] (wie Anm. 7), S. 109 [Übers. geänd.], frz.: Cabanne 1967 (wie Anm. 7), S. 135. Duchamp habe den „alleralltäglichsten Vorgang des Atmens mit Bewusstsein" gefüllt. Kornelia von Berswordt-Wallrabe, „Im Gebrauchsfluß fischen ...", in: Marcel
Duchamp. Respirateur, Ausst.-Kat. Staatliches Museum Schwerin, Ostfildern 1995, S. 11-16, hier: S. 12.

11 Jacques Rancière, „Der Himmel des Plebejers. Paris, 1830“, in: ders. (Hg.), Aisthesis. Vierzehn Szenen, Wien 2013 [frz. 2011], S. 67-84, hier: S. 76.

12 Rancière 2013 [2011] (wie Anm. 11), S. 83.

13 Ebd.

14 Cabanne 1972 [1967] (wie Anm. 7), S. 109 [Übers. geänd.], frz.: Cabanne 1967 (wie Anm. 7), S. 135: "[C]haque seconde, chaque respiration est une œuvre qui n'est inscrite nulle part, qui n'est ni visuelle ni cérébrale."

15 Blunck 2017 (wie Anm. 3), S. 243-247.

16 Serge Stauffer (Hg.), Marcel Duchamp. Die Schriften. Zu Lebzeiten veröffentlichte Texte, Zürich 1994 [1981], S. 125, Nr. 116, frz.: Ecke Bonk/Richard Hamilton (Hg.), Marcel Duchamp: Notes et/and Notations, Köln 2002, S. 21, Nr. 5.

17 Wolfgang Oczenski/Alois Werba/Harald Andel (Hg.), Atmen - Atemhilfen. Atemphysiologie und Beatmungstechnik, Berlin/Wien 2001 [1994], S. 107. 
Atmen als Werk - mithin als etwas, das gemacht wird - tatsächlich, wie Duchamp sagte, „registriert“ werden, insbesondere durch den Rauch von Zigarren. ${ }^{18}$

Im Folgenden wird dargelegt, dass sich Duchamps - zugegeben: sophistisch anmutende - Haltung, die das Atmen als basale, vielleicht sogar als die basalste Form künstlerischer Praxis reklamierte, bereits früh in dessen Denken und überdies in einigen frühen künstlerischen Arbeiten nachweisen lässt. So werde ich im ersten Teil meines Beitrags darlegen, dass und wie Duchamp als junger Künstler in den 1910er Jahren seiner grundsätzlichen Lebenshaltung Ausdruck verlieh - Lebenshaltung verstanden im doppelten Wortsinn: als Existenzwahrung und als Haltung dem Leben gegenüber -, unter anderem indem er das Thema Atemluft gedankenspielerisch reflektierte. Im zweiten Teil werde ich am Beispiel seines Flakons Belle Haleine aus dem Jahr 1921 zeigen, dass Duchamp sich über die avancierten Kunstvorstellungen und die betriebsame Maler-Routine seiner Zeit ironisch mokierte und damit seine - eben mit der Devise des "schönen Atems“ ausgewiesene - Lebenshaltung als eine vollkommen souveräne reklamierte. Atmen war, so wird zu schließen sein, für Duchamp eine Metonymie für das souveräne Dasein.

\section{Luftrationierung und Lebenshaltung}

Es gibt zahlreiche Hinweise darauf, dass der Atem und das Atmen bereits im Frühwerk Duchamps thematisch gewesen sind. Der vielleicht eindrücklichste Beleg findet sich in einer Notiz, die in der ersten Hälfte der 1910er Jahre entstanden sein muss und die Duchamp später, 1934, in seiner sogenannten Grünen Schachtel publizierte (Abb. 1): „Eine Gesellschaft gründen / deren Mitglieder / die Luft bezahlen müssen, die sie atmen / (Luftzähler; Gefangennahme / und verdünnte Luft, Strafe im / Falle des Nichtzahlens / einfache Erstickung bei / Bedarf (die Luft abschneiden)“. ${ }^{19}$ Duchamp formuliert hier die groteske Idee, eines der selbstverständlichsten Allgemeingüter - nämlich die Atemluft - zu kapitalisieren, kontrolliert durch „Luftzähler“ (compteurs d'air). Die geradezu dystopische Idee also, eine theoretisch unendliche natürliche Ressource künstlich zu rationieren und zu kommodifizieren, mit erheblichen Sanktionen im Fall ausbleibender Bezahlung. „Strafe“ (peine),

18 Siehe vertiefend Blunck 2017 (wie Anm. 3), S. 243-247.

19 Stauffer 1994 [1981] (wie Anm. 16), S. 98, Nr. 97, frz.: Bonk/ Hamilton 2002 (wie Anm. 16), S. 20, Nr. 101: „Établir une société dont l'individu ait à / Payer l'air qu'il respire (compteurs d'air); / emprisonnement et air raréfié, peine en / cas de non paiement simple asphyxie au besoin. (couper l'air)“. 


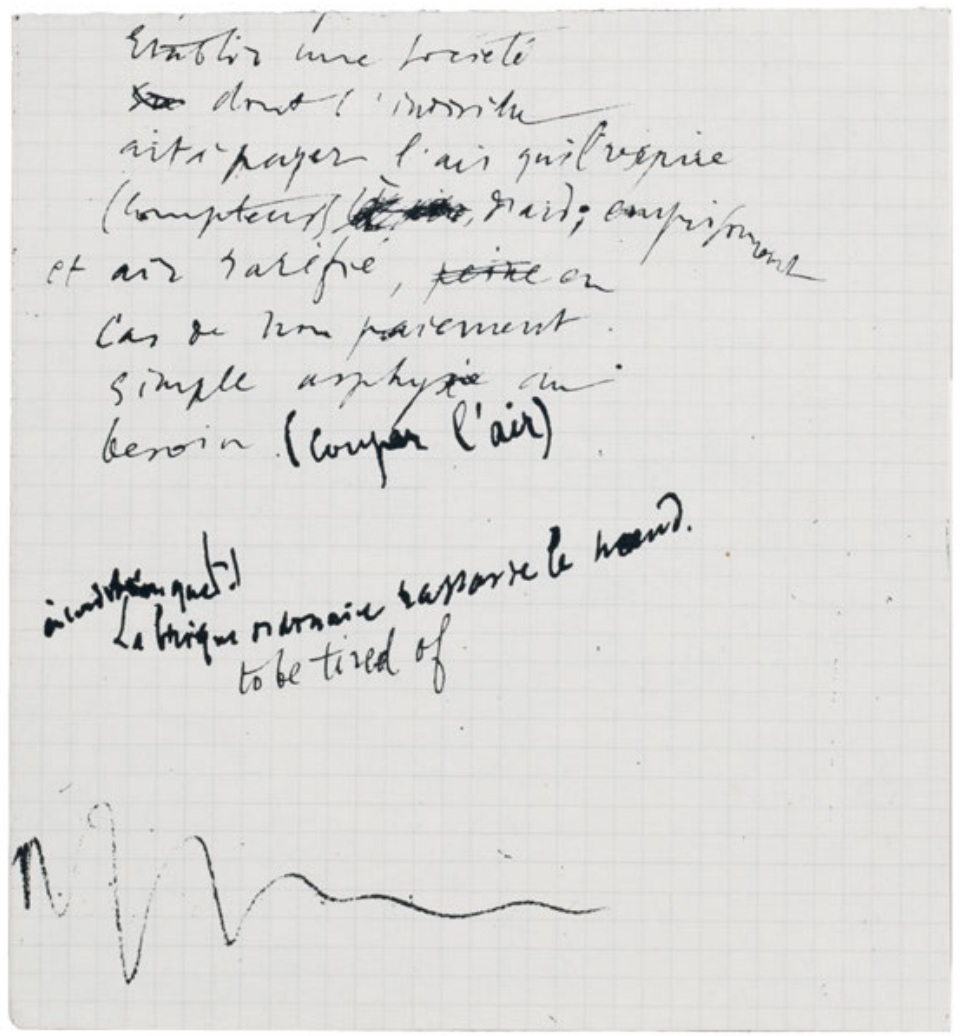

Abb. 1: Marcel Duchamp, handschriftliche Notiz, Tinte auf Papier, ca. 1912-15, 11,6 × 10,5 cm, Centre national d'art et de culture Georges-Pompidou, Paris, reproduziert in: La Mariée mise à nu par ses célibataires, meme, 1934, 94 faksimilierte Notizen, Zeichnungen und Fotografien in Kartonschachtel, Edition in einer Auflage von 320 Exemplaren, $33,2 \times 28,0 \times 2,5 \mathrm{~cm}$ 
schreibt Duchamp zunächst, streicht das Wort jedoch sogleich wieder durch und spezifiziert die drastischen Konsequenzen: Luftverdünnung und sogar „einfache Erstickung bei Bedarf“ beziehungsweise „die Luft abschneiden“ (couper l'air). Ohne Atemluft kein Leben: eigentlich eine Selbstverständlichkeit, in Duchamps dystopischem Szenario jedoch eine ständige Gewissheit und Bedrohung. Zwar wäre einer solchen, eigens zu gründenden Société vermutlich freiwillig beizutreten und dies womöglich unter Anerkenntnis ihrer restriktiven Statuten (was dem Ganzen einen noch absurderen Charakter verliehe). Doch sollte diese Freiwilligkeit nicht verkennen lassen, dass Duchamp hier - wohlgemerkt: gedankenspielerisch - ein Regime im Sinn hat, in welchem Zugang zu und Zahlung von Atemluft über Leben und Tod entscheiden.

Man kann dieses restriktive Regime als „Souveränitätsmacht“20 im Sinne Michel Foucaults bezeichnen. Denn begreift man Duchamps Gedankenspiel wörtlich und zunächst ironiefrei, so findet sich in seiner Dystopie das souveränitätspolitische „Sterben machen“, von dem Foucault später schreiben wird. Die Duchamp vorschwebende Société würde „,auf dem Feld der Souveränität den Tod ausspielen“ ${ }^{21}$ wie Foucault dies für die alte souveränitätspolitische Machttechnik formuliert. Die Sanktionierung des Missbrauchs von Atemluft „macht sterben“: „,einfache Erstickung bei Bedarf“ und „die Luft abschneiden“, benennt Duchamp die Sanktionen, wobei für ihn - anders als später bei Foucault - der Tod nicht Folge einer Strafe, sondern binnenlogische Konsequenz ist; es ist dies wohl der Grund, warum er „peine“ durchstrich. Duchamps Notiz ruft folglich auf, was Foucault die „Todesmacht" 22 nennen wird: einen „Souverän“ (nämlich die zu gründende Société), der „sein Recht über das Leben“23 ausübt, ein Recht, „sterben zu machen und leben zu lassen" ${ }^{24}$ Foucaults berühmte Formel des doppelten Dualismus von Souveränitätsmacht und „Bio-Macht“25 lautet ja: „sterben machen“ und „leben lassen“ (die Souveränitätsmacht) versus „sterben lassen" und „leben machen"

Nun changieren allerdings - eben je nach Lesart - in Duchamps Notiz Souveränitäts- und Biomacht. Denn versteht man Duchamps Szenario nicht bloß in einem buchstäblichen, sondern zugleich in einem

20 Michel Foucault, Der Wille zum Wissen, Frankfurt am Main 1983 [dt. 1977] [frz. 1976], S. 138.

21 Ebd., S. 139.

22 Ebd., S. 132.

23 Ebd.

24 Ebd.

25 Ebd., S. 135. „Biomacht“ als „positive ,Lebensmacht““ ist laut Foucault die „Macht zum Leben“ (S. 134), die sich auf die „Durchsetzung des Lebens" richte. Entsprechend ist von einer mit dem Begriff der Biomacht zusammenhän- genden Biopolitik immer dann zu sprechen, wenn sich, wie Foucault sagt, „das Biologische im Politischen“ reflektiert. Biopolitik unter den Auspizien einer „Normalisierungsgesellschaft“ ziele auf das Leben „verstanden als Gesamtheit grundlegender Bedürfnisse“, auf ein „konkretes Wesen des Menschen“, auf die „Entfaltung seiner Anlagen und [auf die, l.b.] Fülle des Möglichen“. Ebd., S. 132, S. 135 und S. 138-140.

26 Ebd., S. 134. 
übertragenen, eher allegorischen Sinn, so steckt darin der biopolitische Kern eines Bewusstseins dafür, dass Atemluft „leben macht“, ja dass Atmen bloßes Leben ist. 1976 schreibt Foucault in Der Wille zum Wissen (La volonté de savoir) im Anschluss an Aristoteles' Feststellung, der Mensch sei ein „Tier, das auch einer politischen Existenz fähig“"27 sei: „Der moderne Mensch ist ein Tier, in dessen Politik sein Leben als Lebewesen auf dem Spiel steht." ${ }^{28}$ Bereits sechs Jahrzehnte bevor Foucault dies feststellt, hatte Duchamp als junger Mann in den 1910er Jahren in seiner Notiz ein fiktives Szenario ersonnen, in welchem der Mensch sein Leben völlig unnötig aufs Spiel setzt. Er hatte diese Groteske ersonnen mit der gesellschaftspolitischen, ja fast ethischen Botschaft, dass eben dies zugleich in anderen realen Lebensbereichen bereits unhinterfragt geschieht - und zwar ohne dass dazu eine gegebene Notwendigkeit bestünde. Duchamp, so meine Annahme, hatte die ironische Idee einer dystopischen Gesellschaft für bezahlte Atemluft ersonnen, um in einem solchen Gedankenspiel eine Einsicht allegorisch zuzuspitzen: Unter den an sich schon grotesken realen kapitalistischen, autoriativen „Existenzbedingungen“29 ist das Leben fast vollständig kontrolliert und reguliert. Warum dann konsequenterweise nicht auch die Atemluft und damit eben das Leben selbst? ${ }^{30}$

Als Dystopie einer staatlichen Souveränitätsmacht war Duchamps Szenario darauf angelegt, eine Hinterfragung des Grades realer biopolitischer Selbstermächtigung anzuregen. In eine solche Richtung weisend erläuterte Duchamp denn auch völlig unerwartet im Jahr 1958, gut vier Jahrzehnte nach Abfassung seiner Luftrationierungsnotiz, deren Hintergrund: Eine

27 Ebd., S. 138.

28 Ebd.

29 Ebd., S. 137.

30 Mutmaßlich drei Jahrzehnte nach Abfassen seiner Notiz sollte Duchamp erneut ein ironisch-dystopisches, geradezu illiberales Szenario entwerfen. Diesmal allerdings in Form eines Lexikoneintrags und eines dazugehörigen fiktiven Amtsformulars. Das in gegenemanzipatorischem, ordnungs- und obrigkeitsstaatlichem Duktus abgefasste Lemma „Emanzipation“ und dessen doppelseitiges Ergänzungsblatt mit dem Titel „Ein Jahr gültige Lebenserlaubnis" in der sogenannten Da Costa-Enzyklopädie aus dem Jahr 1947 vermeldeten eine alsbald bevorstehende Einführung einer sogenannten „Lebenserlaubnis“, erklärten diese zum „endgültigen Sieg der Demokratie" und feierten diesen permis de vivre als „Krönung einer langen Reihe von Bemühungen, deren Ziel darin bestand, die unveräußerlichen Rechte des Individuums zu festigen und diesem, in den strengen Grenzen seiner Verpflichtungen, eine Freiheit zu sichern, die niemand beeinträchtigen kann“. Der Preis dieser angeblichen Sicherung der Freiheit und der vermeintlichen Festigung individueller Rechte: die Begrenzung der Lebenserlaubnis auf ein Jahr, die allzeitige und will- kürliche Widerrufbarkeit dieser Erlaubnis, eine permanente Ausweis- und Meldepflicht, die allzeit latente Bedrohung mit der Todesstrafe usw. Als angebliche Gewähr individueller Freiheit sollte diese illiberale Einführung einer Lebenserlaubnis der „Regelung der Existenz von Individuen“ dienen, welche der „Kontrolle“ des Staates unterstanden: Individuen, die allzeit mit schwersten Sanktionen rechnen mussten. Beispielsweise: „IV. [...] Jede Person, die, selbst nur auf der Durchreise, in den territorialen Grenzen eines Departments aufgegriffen wird und keine Lebenserlaubnis eben dieses Departments vorweisen kann, hat jegliche Lebenserlaubnis verwirkt. [...] VII. Jeder französische Bürger, der keine Lebenserlaubnis hat oder dessen Lebenserlaubnis nicht ordnungsgemäß ist, muss mit der Todesstrafe rechnen.“ Marcel Duchamp, „Emanzipation“/ „Ein Jahr gültige Lebenserlaubnis“ [frz. 3 Bände, 1947-1949], in: Tom Lamberty/Ronald Voulliè (Hg.), Die Da Costa Enzyklopädie, Berlin 2008, S. 20 und S. 28f. 
„,ideale Gesellschaft würde dem Menschen all die Dinge geben, die für das Leben notwendig sind, doch zur gegenwärtigen Zeit müssen wir Geld verdienen, um zu leben. Diesen Sachverhalt kommentierte ich, als ich den Begriff der Luftrationierung konzipierte. Dies wäre doch ein logisches Ende für die Art und Weise, wie wir jetzt die übrigen dringenden Lebensbedürfnisse rationieren. Die Äußerung über die Luftrationierung war eine boutade - eine scherzhafte Bemerkung -, sie enthielt aber einen Hinweis auf mehr.“31

Die weiteren Ausführungen Duchamps lassen erkennen, worin denn dieses ,Mehr' bestand: Seiner Ansicht nach sei es gar nicht notwendig, dass ein Jeder arbeiten, dass Jede Geld verdienen und dass Alle am monetären Handel mit Gütern - mithin mit Waren - beteiligt sein müssen. ${ }^{32}$ Duchamp stellte die vermeintliche Selbstverständlichkeit der realen Tauschökonomie und der gegenwärtigen Arbeitsgesellschaft radikal in Frage. ${ }^{33}$ Der einzige Anspruch auf Arbeit, den er gelten ließ, betraf die Atemarbeit. „Ich habe immer gedacht“, teilte er 1958 mit,

„dass eigentlich kein Bedürfnis besteht, sein Leben zu verdienen, dass wir dabei nur unsere physischen Probleme vergrößern - mehr nicht. Es wird immer Leute geben, die nicht dasitzen und nachdenken können; diese werden all das tun, was notwendig ist. Sämtliche Dinge, die der Mensch benötigt, sind verfügbar: Weizen, Wein usw. Es ist nur das Problem zu entscheiden, wie sie verteilt werden sollen.“34

Ganz offenkundig war Duchamp kein Anhänger des Kapitalismus, zugleich aber distanzierte er sich explizit von kommunistischen Gesellschafts- und Wirtschaftstheorien. ${ }^{35}$ Duchamp war aus einer radikalliberalen Haltung heraus der Auffassung, dass die Menschen das Recht haben sollten, bloß zu atmen, einfach zu leben, bloß da zu sein, ohne arbeiten zu müssen, ohne etwas machen zu müssen - außer eben zu atmen. Allerdings unterstellte auch Duchamp seine Lebenshaltung einem sich in solchen Überlegungen andeutenden ökonomischen Impe-

31 Stauffer 1992 (wie Anm. 4), S. 66, engl.: Laurence Stephen Gold, „Interview with Marcel Duchamp“, 1958 (Fotokopie eines Typoskripts: Serge Stauffer Archiv, Staatsgalerie Stuttgart), S. i-xxxiii, hier: S. i: „The ideal society would give all things to man that are necessary to life, but at the present time we must earn money to live. I commented on this state of affairs when I conceived the notion of rationing air. This would be the logical end to the way we ration the other necessities of life now. The statement about rationing air was a boutade but it had a suggestion of more."

32 Ebd., S. 66, engl.: ebd., S. i. Dieser Ansicht verlieh er in einem weiteren Gedankenspiel eine anschauliche Gestalt, als er 1964 die Einrichtung eines "hospice des paresseux" vorschlug, eines Heims für Faule: „If you are lazy, and people accept you as doing nothing, you have a right to eat and drink and have shelter and so forth. There would be a home in which you would do all this for nothing. The stipulation would be that you cannot work. If you begin to work you would be sacked immediately." Calvin Tomkins, Marcel Duchamp. The Afternoon Interviews, New York 2013, S. 86f.

33 Siehe auch Tomkins 2013 (wie Anm. 32), S. 88.

34 Stauffer 1992 (wie Anm. 4), S. 66, engl.: Gold 1958 (wie Anm. 31), S. i: „I have always felt that there was no need to earn a living, that by doing so we add to our physical problems - nothing more. There will always be people who cannot sit and think; they will do all that is necessary. All things that man need are available, wheat, wine, etc. It is merely a problem of deciding how to get them distributed.“

35 Siehe Tomkins 2013 (wie Anm. 32), S. 88. 
rativ, der zwar nicht auf monetäre Wertsteigerung angelegt war, aber dennoch einem nutzenmaximierenden Handeln unterlag. Insofern müssen selbst Sympathisant*innen und Duchamp-Verehrer*innen eingestehen, dass Duchamps Denken ironischer Weise Züge dessen trägt, wogegen es sich wendete. Denn auch Duchamp unterzog das Leben einem humankapitaltheoretischen Kalkül. Laut dem Soziologen U1lrich Bröckling würde in humankapitaltheoretischer Sicht - und dies ist auf Duchamp anwendbar - ,jeder Einzelne nicht nur zum Kapitalisten, sondern auch zum Souverän seiner selbst. Mit jeder seiner Handlungen“ würde „er den individuellen Nutzen“ maximieren, „aber auch, um Foucaults Formulierung aufzunehmen, die Macht aus[üben], sich selbst ,leben zu machen[']"..36

Duchamps Idee, sich auf das Atmen zu besinnen und den kapitalistischen Arbeitszwang abzuschaffen, ließ sich jedoch keineswegs mit der sozialen Wirklichkeit vereinbaren, zumal nicht in der Zeit nach dem Zweiten Weltkrieg. So sah sich denn Duchamp im Februar 1963 veranlasst, öffentlich zu beklagen, niemand könne sich heute - und dies eben anders als zu seiner eigenen Jugendzeit - „mehr leisten, ein junger Mensch zu sein, der überhaupt nichts tut. Wer arbeitet schon nicht? Sie können nicht leben, ohne zu arbeiten, was etwas Schreckliches ist." ${ }^{\text {"37 }}$ Als theoretische Gegenposition zur Arbeitspflicht im Kapitalismus rief Duchamp den französischen Sozialisten Paul Lafargue (1842-1911) auf, gleichsam als Kronzeugen seines eigenen Müßiggangs, mit dessen Forderung eines „Rechts auf Faulheit“. Lafargue hatte sich 1880 in seiner Polemik Le Droit à la Paresse gegen das kapitalistische „Dogma der Arbeit" und vor allem gegen die, wie er schrieb, „Arbeitssucht" des Proletariats gewandt und vorgeschlagen, Arbeit durch Rationierung zu reglementieren. Denn wenn Arbeit „weise reglementiert und auf ein Maximum von drei Stunden täglich beschränkt“ würde, könne sie auch für das Proletariat zu einer Beiläufigkeit werden: zu einer „Würze der Vergnügungen der Faulheit“. ${ }^{38}$

Ein solches von Lafargue propagiertes „Recht auf Faulheit“, so nun beklagte Duchamp 1963 ausgerechnet im konsumpreisenden Modemagazin Vogue, ,existiert jetzt nicht mehr. Sie müssen arbeiten, um Ihr Atmen zu rechtfertigen. " ${ }^{39}$ Arbeit als Legitimation des Atmens, das meint hier: Arbeit als Begründung der Existenz, als alleinige Rechtferti-

36 Ulrich Bröckling, „Menschökonomie. Zur politischen Ökonomie des ,nackten Lebens'“', in: Mittelweg 36, 1, Hamburg 2003, S. 3-22, hier: S. 20 .

37 Stauffer 1992 (wie Anm. 4), S. 147, engl.: William C. Seitz, „What's Happened to Art? An interview with Marcel Duchamp on present consequences of New York's 1913 Armory Show", in: Vogue 141 (4), New York, 15. Februar 1963, S. 110, S. 112f. und S. 129-131, hier: S. 129: „You can not afford to be a young man who doesn't do a thing. Who doesn't work? You can't live without working, which is a terrible thing."

38 Paul Lafargue, Das Recht auf Faulheit. Widerlegung des Rechts auf Arbeit von 1848, Frankfurt am Main 2012 [dt. 1883], [frz. 1880], S. 48f. 
gung des Daseins. Die körperliche Grundfunktion der Atmung reicht mithin nicht als Aktivität innerhalb jener Arbeitsgesellschaft, die einen nicht nur materiellen, sondern geradezu arbeitsethischen Legitimationsdruck entfaltet. Duchamp jedoch lehnte die Pflicht zur Arbeit zeitlebens kategorisch ab - zumal die Arbeit als Lebensinhalt -, gerade weil er erkannt hatte, dass der Mensch sich im Zustand der „Zwangsarbeit“ (travaux forcés) ${ }^{40}$ befinde: „Das ist unser Los auf Erden, wir müssen arbeiten, um zu atmen“" ${ }^{41}$ gab er 1964 zu Protokoll. Das Leben sei Zwängen unterworfen, man müsse es gestalten und habe zu arbeiten; zu atmen hingegen bedeute, da zu sein, einfach zu leben, ohne jegliches, wie Duchamp monierte, „Durchbringen“, ohne ein „Durchkommen ", ${ }^{42}$ ohne ein Sich-das-Leben-Verdienen. Wenn, wie der Kunsttheoretiker Helmut Draxler in anderem Zusammenhang bemerkt, die „ultimative Zumutung“ im Leben darin besteht, „dass das Leben gelebt werden muss" ${ }^{43}$ dann strebte Duchamp danach, einfach zu leben, bloß da zu sein, kurzum: zu atmen! Mithin war Atmen für Duchamp eine Manifestation individueller Souveränität und gesellschaftlicher Ungezwungenheit. Atmen war für Duchamp eine Metonymie für das souveräne Dasein. Ein Dasein ohne verbindliche Arbeit, zumindest ohne produktive Arbeit. Doch anders als der italienische Philosoph Maurizio Lazzarato bin ich nicht der Meinung, dass Duchamp eine „Verweigerung der Arbeit" gefordert und die „Logik der Arbeit" ${ }^{44}$ unterwandert hätte. Eine solche Sicht würde Duchamp auf die Position jenes Revoluzzers festlegen, der er nicht war; weniger noch als Herman Melvilles Bartleby hat er sich aktiv entzogen oder agitatorisch gegen etwas gewandt.

\section{Eigenheit und schönes Dasein}

Bereits als junger Mann hatte Duchamp einen radikalen Individualismus entwickelt, der sich auch und insbesondere aus einer Lektüre des Junghegelianers Max Stirner (1806-1856) speiste. Duchamp hatte nachweislich die französische Ausgabe von Stirners 1844 erschienenem Hauptwerk Der Einzige und sein Eigentum gelesen, mehrfach sogar. ${ }^{45}$ Im Zentrum der Philosophie Stirners steht ein radikaler Individualismus,

39 Stauffer 1992 (wie Anm. 4), S. 147, engl.: Seitz 1963 (wie Anm. 37), S. 129: "[...] that right doesn't exist now. You have to work to justify your breathing." Seine individualistische Lebenshaltung offenbarte Duchamp bei verschiedenen Gelegenheiten, beispielsweise 1957, als er rückblickend seine Genügsamkeit herausstrich (fast wie ein Erfolgsrezept seines Lebens): „Nichts von den Dingen, in die das Leben Männer zwängt: Ehefrauen, drei Kinder, ein Landhaus, drei Autos! Ich meide materielle Verpflichtungen." Stauffer 1992 (wie Anm. 4), S. 64, engl. Hellman 1957 (wie Anm. 8), S. 26: „No alimony. Nothing. The things life forces men into - wives, three children, a country house, three cars. I avoid material commitments.“
40 Tomkins 2013 (wie Anm. 32), S. 86.

41 Ebd.: "That's our lot on earth, we have to work to breathe.“

42 Stauffer 1992 (wie Anm. 4), S. 64, engl. Hellman 1957 (wie Anm. 8), S. 26: „get along“.

43 Helmut Draxler, „Die Evolution anpassen. Medien und Avantgarde in biopolitischer Perspektive“, in: Sabeth Buchmann/Helmut Draxler/Stephan Geene (Hg.), Film Avantgarde Biopolitik, Wien 2009, S. 130-153, hier: S. 131.

44 Siehe Maurizio Lazzarato et al., Marcel Duchamp und die Verweigerung der Arbeit, Wien/Linz 2017, S. 10.

45 Siehe u.a. Herbert Molderings, Kunst als Experiment. Marcel Duchamps ,3 Kunststopf-Normalmaße;, München/ Berlin 2006, S. 134-147. 
steht ein Individuum, das sich als ein einzelnes behauptet, sich selbst behauptet, aber auch selbst genießt. Berühmt geworden ist Stirners egozentristische Formel: „Mir geht nichts über Mich!“. ${ }^{46}$ Es nimmt also nicht Wunder, dass Duchamp immer wieder die unbedingte Individualität des Künstlers propagierte und beides, Freiheit und Individualität, auch für sich bedingungslos beanspruchte. Er wolle „frei sein“ ${ }^{47}$ ungebunden, frei sein für sich selbst, so ließ Duchamp immer wieder verlautbaren. Es war seine feste, lebenslange Überzeugung, dass ein Künstler „ungebunden“ und „zur Freiheit bereit" ${ }^{48}$ sein müsse, ohne jegliche Verpflichtungen. Wahre, echte Freiheit aber, dies hatte Duchamp bei Stirner lesen können, bedinge die Erlangung der Eigenheit: ${ }^{49}$ "Welch ein Unterschied zwischen Freiheit und Eigenheit!“, beschwört Stirner seine Leser*innen:
„Gar vieles kann man loswerden, alles wird man doch nicht los, von vielem wird man frei, von allem nicht. [...], Freiheit" lebt man nur in dem ,Reich der Träume!' Dagegen Eigenheit, das ist mein ganzes Wesen und Dasein, das bin ich selbst. [...] Mein eigen bin ich jederzeit und unter allen Umständen, wenn ich mich zu haben verstehe und nicht an andere verwerfe. “50

Eine solche Eigenheit, ein solches „Dasein“ und „Sich-Haben“, fasste Duchamp in der Metonymie des Atmens. Für Duchamp war Atmen die Grundform dessen, was Stirner Eigenheit genannt hatte.

$\mathrm{Zu}$ seiner individualistischen Haltung war Duchamp überdies durch einen ganz konkreten, wie er es später nannte, „Zwischenfall“"51 motiviert worden. Im März 1912 war dem jungen Duchamp eine narzisstische, gerade dadurch jedoch seine weitere künstlerische Entwicklung initiierende Kränkung zugefügt worden. So wurde er von seinem damaligen künstlerischen Umfeld - unter anderem von seinen beiden älteren Brüdern - aus dem Salon des Indépendants gleichsam ,ausjuriert', weil den Kubisten die Bewegungssuggestion in seinem Gemälde Akt eine Treppe hinabsteigend, Nr. 2 (Nu descendant un escalier, $\left.n^{\circ} 2\right), 1912$, missfiel..$^{52}$ Dieser Vorgang sei, erläuterte Duchamp rückblickend, „tatsächlich ein Wendepunkt ${ }^{\text {“53 }}$ in seinem Leben gewesen

46 Max Stirner, Der Einzige und sein Eigentum, Leipzig 1845 [1844], S. 9.

47 Stauffer 1992 (wie Anm. 4), S. 83, engl.: George Heard Hamilton/Richard Hamilton, A Radio Interview, 19. Januar 1959, New York: BBC Radio (maschinenschriftliches Transkript: Serge Stauffer Archiv, Staatsgalerie Stuttgart), o. s. (Blatt 11): „be free“.

48 Ebd., S. 198, engl.: Dore Ashton, „An Interview with Marcel Duchamp“, in: Studio International 878 [171], Juni 1966, S. 244-247, hier: S. 246: „disengaged“ und „ready for freedom".
49 Siehe hierzu auch Maurice Schuhmann, „Stirners Konzept von ,Selbstermächtigung‘. Von der Überwindung der Entfremdung, über die Freiheit hin zur Eigenheit"“, in: Wolf-Andreas Liebert/Werner Moskopp (Hg.), Die Selbstermächtigung der Einzigen. Texte zur Aktualität Max Stirners, Berlin/Münster 2014, S. 67-79.

50 Stirner 1845 [1844] (wie Anm. 46), S. 165f.

51 Cabanne 1972 [1967] (wie Anm. 7), S. 12, frz.: Cabanne 1967 (wie Anm. 7), S. 21: „incident“. 
und hätte ihm „zu einer völligen Befreiung von der Vergangenheit, meiner persönlichen Vergangenheit, verholfen. Ich sagte mir: ,Na, wenn das so ist, dann kommt es nicht in Frage, einer Gruppe [den Kubisten, l.b.] beizutreten, man kann nur mit sich selbst rechnen, man muss allein sein. “"54 Der 24-jährige Duchamp zog sich daraufhin für einige Wochen nach München zurück, sezessionierte sich vom Kubismus und beschloss, zurückgekehrt nach Paris, „aufzuhören, ein Maler im professionellen Sinn zu sein“, ${ }^{55}$ mit der "Welt der Künstler“ sei er fortan "fertig" ${ }^{\prime 6}$ gewesen.

Ausgerechnet mit jenem treppensteigenden Akt nun, der im Frühjahr 1912 zu Duchamps Refüsierung geführt hatte, ist der junge Duchamp, der sich just von konventionellen Künstlertopoi zu emanzipieren beginnt, nur wenige Monate später, im Herbst 1912, im Salon de , la Section d'Or'vertreten. Diese bahnbrechende Kubisten-Ausstellung sollte ihren enormen Erfolg auch einem „wortstarken Rahmenprogramm" ${ }^{57}$ verdanken: einem begleitenden Katalog, insbesondere aber der zeitgleich erscheinenden ersten und einzigen Ausgabe der Zeitschrift La Section d'Or sowie einer Reihe programmatischer Vorträge. ${ }^{58}$ In diesem publizistischen Umfeld der Ausstellung waren es vor allem die Kunstkritiker Guillaume Apollinaire (1880-1918) und Maurice Raynal (1884-1954), die sich als die wortführenden Theoretiker kubistischer Malerei etablierten. ${ }^{59}$ Bereits im Februar 1912 hatte Apollinaire für die neue, kubistische Malerei die formelhafte Bezeichnung der reinen Malerei (peinture pure $)^{60}$ geprägt, in begrifflicher Anlehnung an Stéphane Mallarmés Wort von der poésie pure. In seinem wenig später publizierten Büchlein Les Peintres Cubistes erhob Apollinaire die peinture pure sogar zum normativen Angelpunkt seiner Kunstkritik, insofern er die „reine Malerei“"61 als eine nicht mehr abbildende, somit rein ,malerische', von der Last des Sujets gänzlich befreite, vor allem aber visuell wohlgefällige Malerei konzipierte. Diese „neue“ Malerei würde ein „ungetrübtes ästhetisches Wohlgefallen hervorru-

52 Marcel Duchamp, Nu descendant un escalier $n^{\circ} 2$, Öl auf Leinwand, 1912, $147 \times 89,2 \mathrm{~cm}$, Philadelphia Museum of Art, Sammlung Louise und Walter Arensberg.

53 Stauffer 1992 (wie Anm. 4), S. 180, engl.: Calvin Tomkins, "Marcel Duchamp“, in: ders. (Hg.), The Bride \& the Bachelors. Five Masters of the Avant-Garde, New York 1969 [1965], S. 9-68, hier: S. 22: „really a turning point“.

54 Cabanne 1972 [1967] (wie Anm. 7), S. 38, frz.: Cabanne 1967 (wie Anm. 7), S. 52: ,[...] aidé à me libérer complètement du passé au sens personnel du mot. J'ai dit: ,Bon, puisque c'est comme ça, pas question d'entrer dans un groupe, il ne faudra compter que sur soi, etre seul.“"

55 Stauffer 1992 (wie Anm. 4), S. 180, engl.: Tomkins 1969 [1965] (wie Anm. 53), S. 25: „[...] from 1912 on I decided to stop being a painter in the professional sense".
56 Ebd., S. 138, engl.: Francis Steegmuller, „Duchamp: Fifty Years Later“, in: Show 2 (3), Februar 1963, New York, S. 28-29, hier: S. 28: „world of the artists“ und „through“. 57 Lisa Werner, Der Kubismus stellt aus. Der Salon de la Section d'Or, Paris 1912, Berlin 2011, S. 13.

58 Werner 2011 (wie Anm. 57), S. 13 und S. 233-248.

59 Erwähnt sei überdies die wichtige Publikation zweier führender Kubisten: Albert Gleizes/Jean Metzinger (Hg.), Du Cubisme. On Cubism. Über den Kubismus, Frankfurt am Main 1993 [frz. 1912].

60 Guillaume Apollinaire, Les peintres cubistes. Méditations esthétiques, Paris 2012 [1913], S. 16.

61 Guillaume Apollinaire, „Über den Bildgegenstand in der modernen Malerei“ [frz. 1912], in: Hajo Düchting (Hg.), Apollinaire zur Kunst. Texte und Kritiken 1905-1918, Köln 1989, S. 151-152, hier: S. 151. 


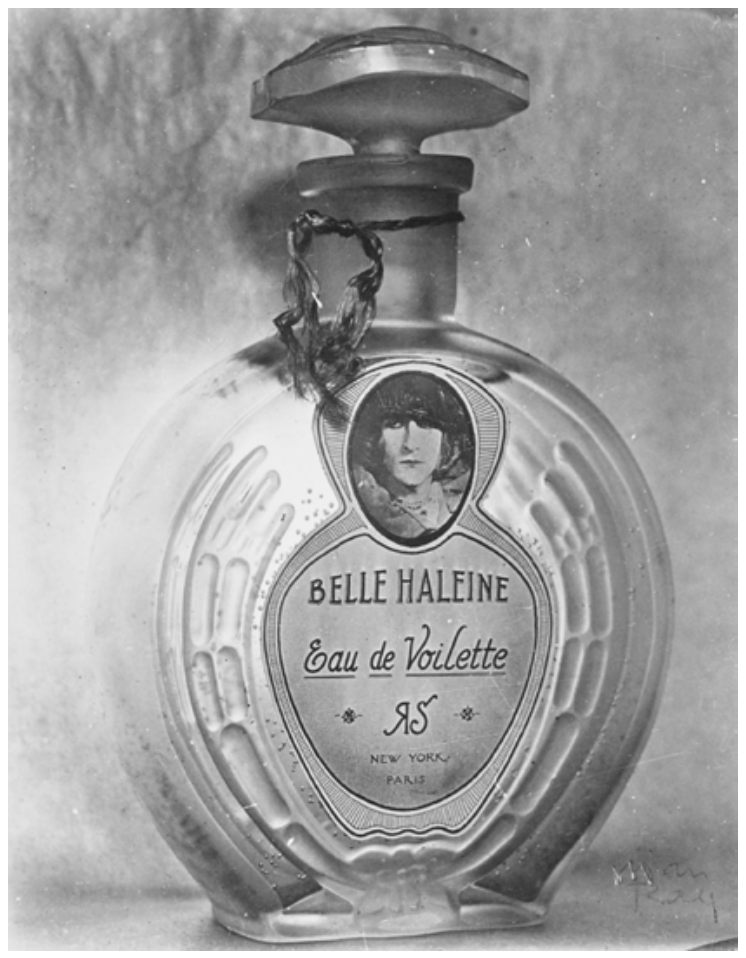

Abb. 2: Marcel Duchamp, Belle Haleine, Parfümflakon mit Etui, 1921, 16,5 × 11,2 cm, Privatsammlung 
fen“, schwärmte Apollinaire und postulierte: „Das ist reine Kunst.“62 Auch Maurice Raynal hatte ab 1912 der peinture pure vehement das Wort geredet und in einem längeren Text in besagter Zeitschrift der Section d'Or jene „einzige Malerei“ (seule peinture) gepriesen, an deren Unübertrefflichkeit er nicht zweifelte: „Gibt es eine schönere Idee als diese Konzeption einer reinen Malerei?“, fragte Raynal 1912 rhetorisch, um umgehend mit seinem Verdikt zu antworten: „Die Malerei soll nichts anderes sein als eine Kunst, die sich aus einem von keinerlei Zwecken bestimmten Studium der Formen herleitet."63

Duchamp nun lehnte eine solche Idee einer reinen Malerei als völlig geistlos ab. Seit 1913 hatte er sich auf eine penible, „metikulöse“ 64 handwerkliche Präzision verpflichtet, auf eine „mechanische Technik“, ${ }^{65}$ eine ,peinture de précision“ ${ }^{66}$ als fundamentale Opposition zur peinture pure. ${ }^{67}$ Rückblickend erklärte er mit großer Zurückhaltung, dass er „die reine Malerei“ für nicht „interessant" Gegenteil: Er habe 1913/14 von der „physische[n] Seite der Malerei“, „Von der Stofflichkeit der Malerei“ ${ }^{69}$ von der „physische[n] Reinheit der Malerei“ (la pureté physique de la peinture), ${ }^{70}$ wegkommen wollen. So habe er die Entscheidung treffen müssen, „[d]ie Malerei, die man die reine nennen könnte, oder die Malerei um ihrer selbst willen, aufzugeben“ und „aus einer festgefahrenen Spur herauszukommen“.71 Dies sei sein „Ausgangspunkt seit 1913 “72 gewesen. Tatsächlich ging Duchamp weiter: Ab 1913 bespöttelte er die peinture pure und ihre Apologeten immer wieder in ironischen Sinnbildern - und dies selbst noch im Jahr 1921,73 als sein enigmatischer Flakon Belle Haleine (Abb. 2) entstand.

62 Guillaume Apollinaire, Die Maler des Kubismus. Ästhetische Betrachtungen, Frankfurt am Main 1989 [frz. 1913], S. 25, frz.: Apollinaire 2012 [1913] (wie Anm. 60), S. 28: „Cet est de l'art pur.“ Man sollte "Sujet" hier allerdings nicht im konventionellen und nachahmenden Sinne als Inhalt missverstehen, also nicht als „eigentliches Sujet“ (S. 13), wie Apollinaire schreibt; andererseits zielte Apollinaire durch seine Verwendung darauf, eben keiner rein abstrakten, völlig gegenstandslosen, bloß formalistischen und formalästhetischen Malerei das Wort zu reden.

63 Maurice Raynal, „Die Ausstellung ,La Section d'Or““ [1912], in: Edward Fry (Hg.), Der Kubismus, Köln 1966, S. 104-107, hier: S. 105; frz.: Maurice Raynal, „L'exposition de ,La Section d'Or'“, in: La Section d'Or 1 (1), 9. Oktober 1912, Paris, o.s. (Blätter 2-5, hier: Bl. 2): „Quelle plus belle idée que cette conception d'une peinture pure [...]. La peinture, en effet, ne doit d'etre qu'un art dérivé de l'étude des formes dans un but désintéressé, c'est-à-dire sans aucun des puis que je viens de citer"

64 Stauffer 1992 (wie Anm. 4), S. 83, engl.: Hamilton/Hamilton 1959 (wie Anm. 47), o.s. (S. 11): „meticulous“.
65 Ebd., S. 55 [Übers. geänd.], engl.: James Johnson Sweeney, „Marcel Duchamp“ [1955], in: James Nelson (Hg.), Wisdom. Conversations with the elder wise men of our day, New York 1958, S. 89-99, hier: S. 95: „mechanical technique".

66 Bonk/Hamilton 2002 (wie Anm. 16), S. 17, Nr. 82.

67 Apollinaire 2012 [1913] (wie Anm. 60), S. 73.

68 Stauffer 1992 (wie Anm. 4), S. 132, frz.: Jouffroy 1964 [1961] (wie Anm. 6), S. 115: „la peinture pure“; „intéressante".

69 Ebd., S. 37, engl.: James Johnson Sweeney, „The Great Trouble with Art in This Country" [1946], in: Michel Sanouillet/EImar Peterson (Hg.), The Writings of Marcel Duchamp, Reprint, New York 1989 [1973], S. 123-126, hier: S. 125: "physical side of painting“; ,physicality of painting“"

70 Ebd., S. 131, frz.: Jouffroy 1964 [1961] (wie Anm. 6), S. 114.

71 Ebd., S. 164, frz.: Jean-Marie Drot, Jeu d'échecs avec Marcel Duchamp, 16mm-Film, 56 min, Frankreich, 1963.

72 Ebd., S. 131, frz.: Jouffroy 1964 [1961] (wie Anm. 6), S. 115: "[...] de me débarrasser de cette idée de peinture pour peinture, ce qui pour moi a été, dès 1913, mon point de départ." 
Dass Duchamp mit Belle Haleine ironisch auf die Ausführungen Raynals des Jahres 1912 in der La Section d'Or reagierte, ist in der Duchamp-Forschung bislang vollkommen unentdeckt geblieben. Raynal hatte den spanischen Kubisten Juan Gris (1887-1927) als den "strengsten Puristen“74 der reinen Malerei gepriesen und dessen Einsatz collagierter Elemente in seinem Gemälde Das Waschbecken (Le Lavabo) von 1912 gepriesen. Gris, so Raynal, sei es mit dem Aufkleben eines Parfümetiketts auf die bemalte Leinwand gelungen, die imitative Darstellung ,glatter Flächen“75 zu vermeiden. Tue man dies nicht, falle man, monierte Raynal, „zurück in die Imitation oder man versucht sich in einer Geschicklichkeit, wie sie der Schildermaler haben muss “ ${ }^{76}$ Die Perspektive des Malers einnehmend fuhr Raynal fort:
„Wenn ich mir einen Flakon vorstelle, und ich will ihn so wiedergeben wie er ist, so erscheint mir sein Etikett als ein nebensächliches Bei- werk, das ich weglassen könnte, denn es ist nichts als ein Abbild. Wenn ich dennoch Wert darauf lege, es darzustellen, könnte ich es genau kopieren. Dies aber wäre eine unnötige Arbeit, denn ebenso gut kann ich das wirkliche Etikett auf das Bild kleben [...]."77

Eben dies, so Raynal, hätte Gris 1912 in und mit seinem Gemälde Le Lavabo geleistet.

Duchamp nun bewerkstelligte ein knappes Jahrzehnt später, 1921, mit Belle Haleine - und ich meine: sehr bewusst und als verspätete, ironische Replik auf die Theoriebildungen der peinture pure - exakt das Gegenteil dessen, was Raynal am Beispiel des Gris-Gemäldes gefordert hatte: Während Raynal monierte, konventionelle Malerei würde sich fehlgängiger Weise um die bildliche Imitation eines Flakons mühen und vorschlug, einfach ein "véritable étiquette“ auf die Leinwand zu kleben, verwendete Duchamp einen realen Flakon aus geschliffenem Glas und klebte darauf ein falsches, da simuliertes Etikett. Während Raynal die „Geschicklichkeit“ (l'habileté) der "Schildermaler“ (peintres d'enseignes) diskreditierte und während er die Nachahmung eines solchen Etiketts zur „travail inutile“ erklärte, erschien Duchamp die Herstellung eines Etiketts gerade als lohnenswerte Tätigkeit und

73 Siehe Lars Blunck, „La Bagarre d'Austerlitz. Ein ironisches Memorial“, in: Marcel Duchamp. 100 Fragen. 100 Antworten, Ausst.-Kat. Staatsgalerie Stuttgart, München/London/New York 2018, S. 108-115.

74 Raynal 1966 [1912] (wie Anm. 63), S. 106 [Übers. geänd.], frz.: Raynal 1912 (wie Anm. 63), o.S. (S. 4): „les plus farouche des puristes".

75 Ebd., frz.: ebd.: „surface planes“.
76 Ebd.: ,[...] si on le fait nous retombons dans l'imitation ou dans la recherche de l'habileté qui spécialise les peintres d'enseignes."

77 Ebd., frz.: ebd.: „Cependant si je tiens à la faire figurer, je pourrais la copier exactement, mais c'est un vrai travail inutile, aussi bien je pose la véritable étiquette sur le tableau après l'avoir cependant découpée suivant la forme que j'ai donnée au flacon [...].“ 
ließ bei der Anfertigung eben dieses Etiketts durch seinen Freund Man Ray eine eben solche „habileté“ an den Tag legen. Und während Raynal das Etikett zum „accessoire négligeable“ und zum bloßen „Ebenbild“ (image) erklärte, erhob Duchamp das Etikett zum alleinigen künstlerischen Arbeitsfeld und nutzte es geschickt für seine, sogleich abschließend noch genauer zu erfassende Mokerie. Es sind dies lauter Inversionen, die den Schluss nahelegen, dass Duchamp 1921 mit Belle Haleine, wie zuvor schon mit anderen Arbeiten wie beispielsweise Die Balgerei von Austerlitz (La Bagarre d'Austerlitz) von $1921^{78}$ ironisch-mokant auf eine ihm unliebsame Kunstpublizistik des Jahres 1912 rekurrierte.

Es mag Duchamp selbst gewesen sein, der sich für Belle Haleine - vielleicht während eines mehrmonatigen Aufenthalts in Paris in der zweiten Jahreshälfte 1921 - den handelsüblichen Parfümflakon des Pariser Parfümiers Rigaud angeeignet hatte. Ansonsten jedoch hatte Duchamp es bei einer rein konzeptuellen Arbeit belassen, insofern er seinen Freund Man Ray die anspruchsvolle, handwerkliche Anfertigung des Etiketts übertragen hatte. Man Ray (1890-1976) hatte eine linear-grafische Kartusche im Stil des frühen Art déco entworfen. Deren oberes, ovales Feld ist - dort wo auf dem originalen Rigaud-Etikett ein bekröntes „R“79 prangt - mit einem Porträt von Duchamps Alter Ego besetzt: eine mysteriöse Dame mit dem lautmalerischen Namen Rrose Sélavy. Auf diese Markenfigur ${ }^{80}$ des fiktiven Parfüms wird auch im unteren, schildartigen Feld der Kartusche angespielt mittels des Monogramms „RS“ (an Stelle des Markenschriftzugs „Rigaud“), flankiert von zwei grafisch stilisierten Blumenminiaturen und gefolgt von den Ortsangaben „New York“ und „Paris“ (Duchamps und damit auch Sélavys zwei Hauptlebensstationen). Grafisch präsenter jedoch noch sind die Schriftzüge „BELLE HALEINE“ und „Eau de Voilette“ (statt ursprünglich „UN AIR EMBAUMÉ“), die mehrere, ambivalente semantische Bedeutungen haben. So bedeutet das facettenreiche Wortspiel „Belle Haleine“ wörtlich „Schöner Atem“, ganz so als diene die im Flakon zu erwartende Flüssigkeit - und wir wissen nicht, ob der heute leere Flakon überhaupt jemals eine solche enthielt - als Mundwasser und somit einer kosmetischen Schönung des Atems. Zugleich aber kann

78 Marcel Duchamp, La Bagarre d'Austerlitz, Miniaturfenster, vorderseitig grau bemalt, 1921, 62,8 × 28,7 × 6,3 cm (Holzsockel: $33,0 \times 20,2 \times 5,0 \mathrm{~cm}$ ), Staatsgalerie Stuttgart.

79 Rigaud inszenierte bei späten Auflagen des Parfüms 'UN AIR EMBAUMÉ' mittels dieses „R“ ein Wortspiel: Einerseits steht das fast royal gestaltete "R“ unverkennbar als Initiale für Rigaud; zugleich wird es bei einigen Flakonetiketten umgeben von „Un“ oben und „Embaumé“ unten, sodass das „R“ als „air“ und somit als Teil des Produktnamens lesbar ist.
80 Helen Molesworth hat Rrose Sélavy als Duchamps „trade mark" identifiziert und ihr eine authentifizierende Funktion zugeschrieben. Siehe Helen Molesworth, „Rrose Sélavy goes Shopping“, in: Leah Dickerman/Matthew S. Witkovsky (Hg.), The Dada Seminars, Washington DC 2005, S. 173-189, hier: S. 181. 
im Markensignet „BELLE HALEINE“ eine Anspielung auf Jacques Offenbachs, 1864 in Paris uraufgeführte opéra-bouffe La Belle Hélène erkannt werden. Der freigeistige Komponist Offenbach (1819-1880) hatte den antiken Mythos der schönen Helena aktualisiert und parodistisch ins Zweite Kaiserreich übertragen, um recht gewitzt bürgerliche Moralvorstellungen vorzuführen, ${ }^{81}$ eine Haltung, der Duchamp durchaus Sympathien entgegen gebracht haben dürfte. Hingegen mag die Produktbezeichnung „Eau de Voilette“ - in Vertauschung der Vokale i und $\mathrm{o}-$ mit dem Begriff eau de violette spielen, einem Veilchenwasser, das heißt einem handelsüblichen, alkoholfreien Aromawasser, was allerdings nicht recht mit der Gefäßform eines Parfümflakons zusammengeht. Vermutlich wird somit eher ein Eau de toilette konnotiert, also ein alkoholbasiertes Duftwasser (von erheblich geringerer Duftkonzentration allerdings als ein klassisches Parfüm). Überdies aber mag „Eau de Voilette“, wörtlich verstanden als ein "Schleierwasser“ (vom Verb voiler: verschleiern, verbergen, verhüllen), eine Verschleierung indizieren: Vielleicht die Verschleierung eines Eigengeruchs, welcher Art dieser auch immer sein möge; vermutlich eher jedoch noch die Verschleierung einer Identität, einer Autorschaft, vielleicht sogar der Künstlerschaft Duchamps, auch wenn der Künstler Marcel Duchamp durch die Travestie als fiktive Markenfigur Rrose Sélavy immer noch durchscheint. Jedenfalls lässt sich „Voilette“ gut auf Duchamps Handlung beziehen, sich als Frau zu verkleiden, sich zu maskieren, sich eben $\mathrm{zu}$,verschleiern ${ }^{682}$ und sich dergestalt einer konventionellen Künstlertopik zu entziehen, mit der Duchamp nach 1912 nachhaltig gebrochen hatte.

Insofern dürfte der Titel Belle Haleine im Sinne von ,Schöner Atem“ für Duchamp auch das schöne, da angenehme, da tätigkeitsarme, mühelose, eben arbeitsarme Dasein aufgerufen haben: eine völlig andere, neue Konzeption von Künstlerschaft. Ein Dasein ohne manifeste Arbeit, einzig mit der Arbeit der Atmung, ein Leben, das buchstäblich bestimmt ist allein von einer Form von Arbeit: der Atemarbeit. Ob es eine „schönere Idee als diese Konzeption einer reinen Malerei“"83 gebe, hatte Maurice Raynal 1912 rhetorisch im Vorfeld seiner Ausführungen zur Collage eines Flakonetiketts bei Juan Gris gefragt. 1921 antwortete

81 Siehe Hans-Jörg Neuschäfer, „Die Mythenparodie in La Belle Hélène“, in: Elisabeth Schmierer ( $\mathrm{Hg}$.), Jacques Offenbach und seine Zeit, Laaber 2009, S. 172-182, hier: S. $173 f$.

82 Vgl. Caro Verbeek, „Historisches Intermezzo. Air de Marcel“, in: Es liegt was in der Luft! - Duft in der Kunst, Ausst.-Kat. Museum Villa Rot, Burgfrieden-Rot 2015, S. 24-41, hier: S. 29; Caro Verbeek, „Surreale Aromen - Zur (Re-)Konstruktion des flüchtigen Erbes von Marcel Duchamp“, in: Museum Tinguely, Basel (Hg.), Belle Haleine. Der Duft der Kunst. Interdisziplinäres Symposi- um, Heidelberg/Berlin 2015, S. 115-125, hier: S. 117. Allerdings akzentuiert Verbeek ihre Interpretation gänzlich anders, wenn sie ebenda schreibt: „Duchamps Kunstwerk schreibt sich in das Themenfeld von Verführung und sexueller Erregung ein, und so liegt die Vermutung nahe, dass er uns auf die unterschätzte Rolle der Nase aufmerksam machen möchte, wenn es um sexuelle Identität geht."

83 Raynal 1966 [1912] (wie Anm. 63), hier: S. 105, frz.: Raynal 1912 (wie Anm. 63), o.s. (S. 2): „plus belle idée que cette conception d'une peinture pure“. 
Duchamp mit der ihm eigenen Verspätung: Ja, den schönen Atem, das bloße, souveräne Dasein. Wenn Atmen für ihn die Tätigkeit des Untätigen war, dann war das schöne Atmen somit der Genuss souveräner Untätigkeit. 
